\title{
Option Pricing When Changes of the Underlying Asset Prices Are Restricted
}

\author{
George J. Jiang ${ }^{1,2}$, Guanzhong Pan $^{2}$, Lei Shi ${ }^{3}$ \\ ${ }^{1}$ University of Arizona, Tucson, USA \\ ${ }^{2}$ College of Finance, Yunnan University of Finance and Economics, Kunming, China \\ ${ }^{2}$ College of Statistics and Mathematics, Yunnan University of Finance and Economics, Kunming, China \\ Email: gjiang@email.arizona.edu \\ Received April 8, 2011; revised June 13, 2011; accepted July 10, 2011
}

\begin{abstract}
Exchanges often impose daily limits for asset price changes. These restrictions have a direct impact on the prices of options traded on these assets. In this paper, we derive closed-form solution of option pricing formula when there are restrictions on changes in underlying asset prices. Using numerical examples, we illustrate that very often the impact of such restrictions on option prices is substantial.
\end{abstract}

Keywords: Option Pricing, Restrictions on Asset Price Changes, Numerical Illustration

\section{Introduction}

Conventional option pricing models assume that there are no restrictions on changes of underlying asset prices. For example, [1,2] specify that stock prices follow a geometric Brownian motion and stock returns follow a normal distribution. Based on a portfolio replication strategy or equivalently the risk-neutral method, option prices are derived as expected payoff of the contract under the risk-neutral distribution, discounted by the riskfree rate.

In practice, however, asset price changes may subject to restrictions imposed by exchanges. For example, CBOT (Chicago Board of Trade) and CME (Chicago Mercantile Exchange) both have daily price limits for futures contracts except currency futures. Daily price limit serves as a precautionary measure to prevent abnormal market movement. The price limits, quoted in terms of the previous or prior settlement price plus or minus the specific trading limit, are set based on particular product specifications. For example, the current limit of daily price changes on short term corn futures is $4.5 \%$. In 1996, Chinese stock market also introduces restrictions on daily stock price changes. Specifically, except the first trading day of newly issued stocks, the limit of stock price change in a trading day relative to previous day's close price is $10 \%$, and for stocks that begin with $\mathrm{S}$, ST, S*ST letters, the limit is $5 \%$. It is clear that such restrictions reduce the value of options since extreme returns on a daily level are ruled out. Nevertheless, how to evaluate the prices of option contracts when changes on underlying asset prices are restricted? How much is the exact impact of such daily price limits on option prices? These questions are yet to be examined in the extant literature.

In this paper, we first derive the option pricing formula when there are restrictions on daily changes of underlying asset prices. We perform the analysis under the Black-Scholes-Merton model framework. Then, we provide numerical comparisons between option prices with and without restrictions on underlying asset price changes.

\section{Option Pricing with Restrictions on Underlying Asset Price Changes}

\subsection{Risk-Neutral Valuation under the Black and Scholes [1] and Merton [2] Framework}

In this section, we first review the risk-neutral approach of option pricing under Black-Scholes-Merton framework. The same approach will be used in the next subsection to derive option pricing formula when there are restrictions on underlying asset price changes. Black and Scholes [1] and Merton [2] assume that stock price $S_{t}$ follows a geometric Brownian motion:

$$
\mathrm{d} S_{t}=\mu S_{t} \mathrm{~d} t+\gamma S_{t} \mathrm{~d} W_{t}
$$

where $\mu$ and $\gamma$ are expected return and volatility, $W_{t}$ is a standard Brownian motion. It is also assumed that the continuously compounded risk-free interest rate, 
denoted by $r$, is constant. The key feature in BlackScholes-Merton framework is that asset return volatility is constant and market is complete. As such, in a riskneutral world, expected return of the underlying stock is equal to risk-free interest rate. That is,

$$
\mathrm{d} S_{t}=r S_{t} \mathrm{~d} t+\gamma S_{t} \mathrm{~d} W_{t}^{Q} .
$$

where $W_{t}^{Q}$ is a standard Brownian motion under the risk-neutral probability measure $Q$.

Consider a European call option with strike price $K$ and maturity $T$ measured in the number of trading days. The price of such option can be computed as

$$
C=\mathrm{e}^{-r T \Delta} E_{Q}\left[\max \left(S_{T}-K, 0\right)\right],
$$

where $E_{Q}[\cdot]$ indicates expectation under risk-neutral measure $Q$, and $\Delta$ is the time interval of a trading day. As shown in many derivatives textbooks, for example [3], the option pricing formula is given as:

$$
C=S_{0} \Phi\left(d_{1}\right)-\mathrm{e}^{-r T \Delta} K \Phi\left(d_{2}\right),
$$

where $\Phi(x)=\int_{-\infty}^{x} \frac{1}{\sqrt{2 \pi}} \mathrm{e}^{-\frac{u^{2}}{2}} \mathrm{~d} u$ is the cumulative distribution function (cdf) of standard normal distribution, and

$$
\begin{gathered}
d_{1}=\frac{\ln \left(S_{0} / K\right)+\left(r+\frac{1}{2} \gamma^{2}\right) T \Delta}{\gamma \sqrt{T \Delta}}, \\
d_{2}=d_{1}-\gamma \sqrt{T \Delta} .
\end{gathered}
$$

This is the famous Black-Scholes-Merton option pricing formula. By constructing a riskless portfolio with option and underlying stock and based on no arbitrage argument, [1] and [2] derive the above option pricing formula as a solution to a partial differential equation (PDE).

\subsection{Closed-Form Option Pricing Formula with Restrictions on Underlying Asset Price Changes}

As mentioned in the introduction, many exchanges impose restrictions on daily price changes of traded assets. As a result, the range of asset return (in logarithmic form) is no longer $(-\infty, \infty)$, but truncated from both below and above. The restriction is particularly important in option pricing since the tail behavior of asset returns has a significant effect on the payoff of options contracts.

Let us start with a normal random variable $X \sim \mathcal{N}$ $\left(\mu, \sigma^{2}\right)$ with probability density function:

$$
f(x)=\frac{1}{\sqrt{2 \pi} \sigma} \mathrm{e}^{-\frac{(x-\mu)^{2}}{2 \sigma^{2}}} .
$$

In addition, $a$ and $b$ are two positive constants. Truncating the left tail of the normal density by $a$ below the mean, and the right tail by $b$ above the mean, the truncated normal distribution is illustrated in Figure 1. Normalizing the truncated density function to make sure the total probability is equal to 1 , we obtain the pdf of a truncated normal random variable $X \sim \mathcal{N}_{\text {trc }}\left(\mu, \sigma^{2}\right)$,

$$
f_{\text {trc }}(x)=\frac{1}{c} f(x), x \in[\mu-a, \mu+b],
$$

where $f(x)$ is the normal density function and

$$
c=\Phi\left(\frac{b}{\sigma}\right)+\Phi\left(\frac{a}{\sigma}\right)-1,
$$

where $\Phi(\cdot)$ is the cdf of standard normal distribution. In practice, price restrictions are often imposed in terms of daily simple returns. For example, daily simple returns in absolute value are restricted to be less than $\delta$, then for $\log$ returns, these restrictions are $a=-\ln (1-\delta)$ and $b=\ln (1+\delta)$.

For the purpose of option pricing, it is also convenient to obtain the characteristic function $(\mathrm{CF})$ of stock returns. As derived in the Appendix, the characteristic function of the truncated normal variable $X$ is given by:

$$
\psi_{\text {trc }}(\omega)=\frac{c^{\prime}(i \omega)}{c} \psi(\omega),
$$

where $\psi(\omega)=\mathrm{e}^{\mu i \omega-\frac{1}{2} \sigma^{2} \omega^{2}}$ is the CF of a normal random variable and

$$
c^{\prime}(i \omega)=\Phi\left(\frac{b}{\sigma}-\sigma i \omega\right)+\Phi\left(\frac{a}{\sigma}+\sigma i \omega\right)-1 .
$$

Since limits are typically imposed on daily price changes and option maturity can be more than one day, we need to derive the distribution of returns over multiple days. Denote $X_{t}=\ln S_{t}$ as the $\log$ price, $Y_{t}=\ln S_{t}-\ln S_{t-\Delta}$ as the daily $\log$ return. Suppose we have $T$ trading days, $1, \cdots, T$, and the daily log returns are iid truncated normal random variables, i.e., $Y_{t} \sim \operatorname{iid} \mathcal{N}_{t r c}\left(\mu, \sigma^{2}\right)$. The $\log$ price at the end of day $T$ is

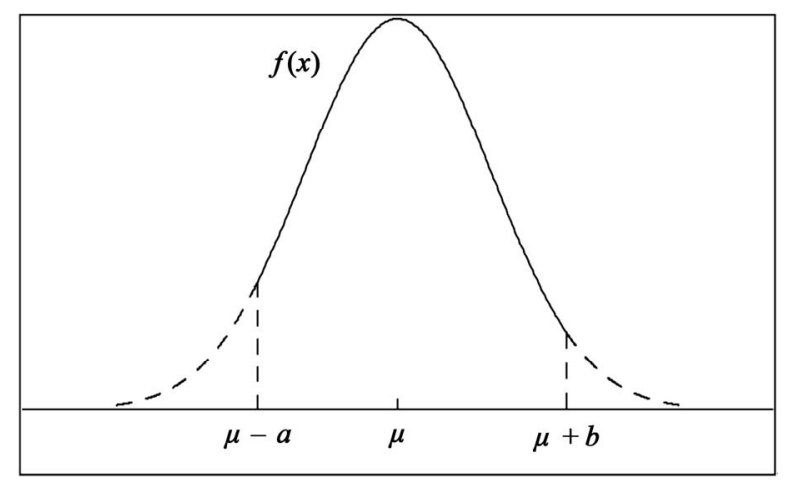

Figure 1. Truncating normal density. 


$$
X_{T}=X_{0}+\sum_{t=1}^{T} Y_{t}, Y_{t} \sim \operatorname{iid} \mathcal{N}_{\operatorname{trc}}\left(\mu, \sigma^{2}\right) .
$$

Similarly, as derived in the Appendix, the CF of $X_{T}$ is given by

$$
\psi_{X_{T}}(\omega)=\mathrm{e}^{i \omega X_{0}}\left[\frac{c^{\prime}(i \omega)}{c} \psi(\omega)\right]^{T} .
$$

In the following, we follow the same risk-neutral approach as outlined in the previous subsection to price options when there are restrictions on underlying asset price changes. As seen in the Black-Scholes-Merton framework, when we move from real world into riskneutral world, volatility remains the same, but expected return is equal to risk-free interest rate. Option prices are then calculated as expected payoff under the risk-neutral measure, further discounted by risk-free interest rate. In the following, we first derive the risk-neutral distribution of asset returns when daily returns follow truncated normal distributions, and then derive a closed-form formula for European call options.

Lemma Let $Y_{t} \sim$ iid $\mathcal{N}_{t r c}\left(\mu, \sigma^{2}\right), t=1, \cdots, T$ and the time interval between observations is $\Delta$, we have

i) Under the risk-neutral measure $Q$ where the expected return of the asset is given by the risk-free rate $r$, we have $Y_{t} \sim$ iid $\mathcal{N}_{t r c}\left(\theta, \sigma^{2}\right), t=1, \cdots, T$, with

$$
\theta=r \Delta-\frac{1}{2} \sigma^{2}-\ln \left[\frac{c^{\prime}(1)}{c}\right] \text {. }
$$

ii) The price of a European call option with strike price $K$ and maturity $T$ is given by

$$
C_{\text {trc }}=S_{0} P_{1}\left(X_{T}>\ln K\right)-\mathrm{e}^{-r T \Delta} K P_{2}\left(X_{T}>\ln K\right),
$$

The probabilities $P_{1}$ and $P_{2}$ can be computed numerically as

$$
P(X>x)=\frac{1}{2}+\frac{1}{\pi} \int_{0}^{\infty} \operatorname{Re}\left[\frac{\exp (-i \omega x) \varphi(\omega)}{i \omega}\right] \mathrm{d} \omega,
$$

where $\varphi(\omega)$ is the CF corresponding to $P$.

Proof: i) Let $\Delta$ be the time interval of each trading day, and the fact that expected return is equal to risk free rate leads to:

$$
E_{Q}\left[S_{T}\right]=\mathrm{e}^{r T \Delta} S_{0}
$$

From the moment generating function of a truncated normal distribution as derived in the Appendix, we have

$$
E_{Q}\left[S_{T}\right]=E_{Q}\left[\mathrm{e}^{X_{T}}\right]=S_{0}\left[\frac{c^{\prime}(1)}{c} \mathrm{e}^{\theta+\frac{1}{2} \sigma^{2}}\right]^{T},
$$

From the above two equations, we have the expression for $\theta$.

ii) According to risk-neutral pricing method, the price of a European call option with strike price $K$ and maturity $T$ is

$$
\begin{aligned}
C & =\mathrm{e}^{-r T \Delta} E_{Q}\left[\max \left(S_{T}-K, 0\right)\right] \\
& =\mathrm{e}^{-r T \Delta} E_{Q}\left[\max \left(\mathrm{e}^{X_{T}}-K, 0\right)\right] \\
& =\mathrm{e}^{-r T \Delta} \int_{\ln K}^{\infty}\left(\mathrm{e}^{x}-K\right) f_{X_{T}}(x) \mathrm{d} x \\
& =\mathrm{e}^{-r T \Delta} \int_{\ln K}^{\infty} \mathrm{e}^{x} f_{X_{T}}(x) \mathrm{d} x-K \mathrm{e}^{-r T \Delta} \int_{\ln K}^{\infty} f_{X_{T}}(x) \mathrm{d} x .
\end{aligned}
$$

where $f_{X_{T}}(x)$ is the pdf of log price $X_{T}$ at time $T$. In addition, under the risk-neutral measure,

$$
\begin{aligned}
S_{0} & =\mathrm{e}^{-r T \Delta} E_{Q}\left[S_{T}\right]=\mathrm{e}^{-r T \Delta} E_{Q}\left[\mathrm{e}^{X_{T}}\right] \\
& =\mathrm{e}^{-r T \Delta} \int_{-\infty}^{\infty} \mathrm{e}^{y} f_{X_{T}}(y) \mathrm{d} y
\end{aligned}
$$

So we have

$$
\mathrm{e}^{-r T \Delta}=\frac{S_{0}}{\int_{-\infty}^{\infty} \mathrm{e}^{y} f_{X_{T}}(y) \mathrm{d} y} .
$$

Substituting this into option price $C$, we get

$$
C=S_{0} \int_{\ln K}^{\infty} \frac{\mathrm{e}^{x} f_{X_{T}}(x)}{\int_{-\infty}^{\infty} \mathrm{e}^{y} f_{X_{T}}(y) \mathrm{d} y} \mathrm{~d} x-\mathrm{e}^{-r T \Delta} K \int_{\ln K}^{\infty} f_{X_{T}}(x) \mathrm{d} x,
$$

Denote

$$
g(x)=\frac{\mathrm{e}^{x} f_{X_{T}}(x)}{\int_{-\infty}^{\infty} \mathrm{e}^{y} f_{X_{T}}(y) \mathrm{d} y} .
$$

Since $\int_{-\infty}^{\infty} g(x)=1, g(x)$ is a pdf. Therefore, we can write the European call option price as

$$
C=S_{0} P_{1}\left(X_{T}>\ln K\right)-\mathrm{e}^{-r T \Delta} K P_{2}\left(X_{T}>\ln K\right) .
$$

$\diamond$ End of proof.

As shown in [4] that the probabilities in (8) can be computed numerically by their corresponding CFs as follows. From the Fourier inversion, we have

$$
P(X>x)=\frac{1}{2}+\frac{1}{\pi} \int_{0}^{\infty} \operatorname{Re}\left[\frac{\exp (-i \omega x) \psi(\omega)}{i \omega}\right] \mathrm{d} \omega .
$$

To compute the CF corresponding to $g(x)$, denoted as $\psi_{1}(\omega)$, by definition, we have

$$
\begin{gathered}
\psi_{1}(\omega)=\int_{-\infty}^{\infty} \mathrm{e}^{i \omega x} g(x) \mathrm{d} x=\int_{-\infty}^{\infty} \mathrm{e}^{i \omega x} \frac{\mathrm{e}^{x} f_{X_{T}}(x)}{\int_{-\infty}^{\infty} \mathrm{e}^{y} f_{X_{T}}(y) \mathrm{d} y} \mathrm{~d} x \\
=\frac{\int_{-\infty}^{\infty} \mathrm{e}^{(i \omega+1) x} f_{X_{T}}(x) \mathrm{d} x}{\int_{-\infty}^{\infty} \mathrm{e}^{y} f_{X_{T}}(y) \mathrm{d} y}
\end{gathered}
$$

where the numerator is given by

$$
\begin{aligned}
& \int_{-\infty}^{\infty} \mathrm{e}^{(i \omega+1) x} f_{X_{T}}(x) \mathrm{d} x=M_{X_{T}}(i \omega+1) \\
& =\mathrm{e}^{(i \omega+1) X_{0}}\left[\frac{c^{\prime}(i \omega+1)}{c} M((i \omega+1) ; \theta, \sigma)\right]^{T} .
\end{aligned}
$$


and the denominator is given by

$$
\int_{-\infty}^{\infty} \mathrm{e}^{y} f_{X_{T}}(y) \mathrm{d} y=M_{X_{T}}(1)=\mathrm{e}^{X_{0}}\left[\frac{c^{\prime}(1)}{c} M(1 ; \theta, \sigma)\right]^{T}
$$

Hence,

$$
\begin{gathered}
\psi_{1}(\omega)=\frac{\mathrm{e}^{(i \omega+1) X_{0}}\left[\frac{c^{\prime}(i \omega+1)}{c} M((i \omega+1) ; \theta, \sigma)\right]^{T}}{\mathrm{e}^{X_{0}\left[\frac{c^{\prime}(1)}{c} M(1 ; \theta, \sigma)\right]^{T}}} \\
=\mathrm{e}^{i \omega X_{0}}\left[\frac{c^{\prime}(i \omega+1) M((i \omega+1) ; \theta, \sigma)}{c^{\prime}(1) M(1 ; \theta, \sigma)}\right]^{T} .
\end{gathered}
$$

\subsection{Numerical Illustrations}

In this section, we illustrate numerically the differences of option prices with and without restrictions on underlying asset price changes.
Table 1 reports the differences in European call option prices under different scenarios. The Black-ScholesMerton price, denoted by CallBSM, is the call option price without price restriction and is computed from formula (3), the call option price with price restriction, denoted by CallTre, is computed from formula (8) derived in the previous subsection.

In Panel A, we set the price limit as $\delta=4.5 \%$, consistent with the current limit of daily price changes on short term corn futures at CBOT, initial stock price $S_{0}=\$ 100$, strike price $K=\$ 100$, maturity $T=10$ days, annualized risk-free interest rate $r=5 \%$. The annualized volatility ranges from $15 \%$ to $50 \%$. As expected, option prices with restricted changes in underlying asset prices are lower than the Black-Scholes-Merton price. The relative difference is higher as volatility increases. For the at-the-money option considered, the relative difference is $19.45 \%$ when volatility is $40 \%$ which is typical for individual stock returns.

Table1. European Call Option Prices with and without Restrictions on Asset Price Changes.

Panel A: Variation in Volatility $\sigma$

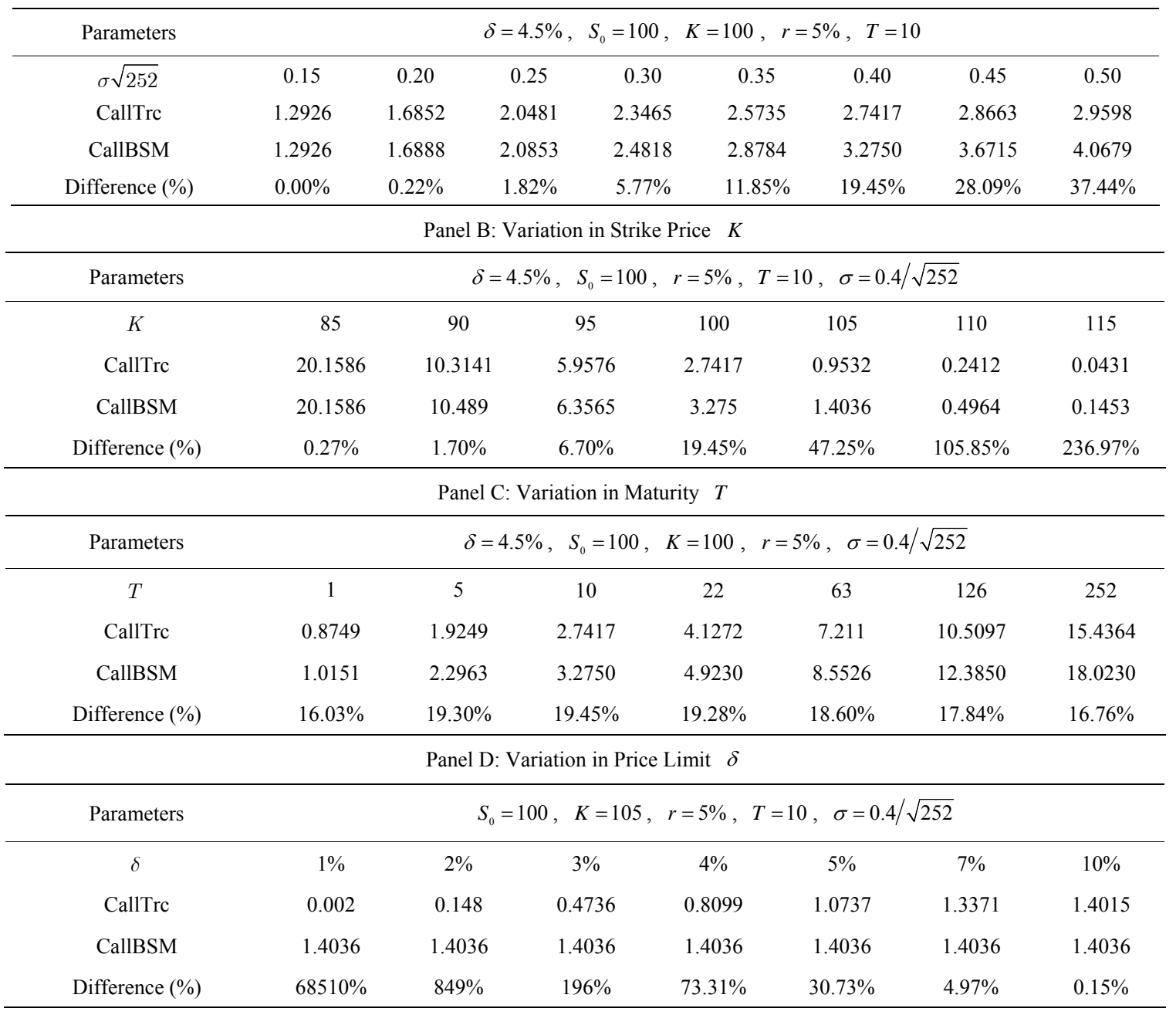


As expected, when the option strike price is higher, the relative difference also increases. The results are illustrated in Panel B where volatility is set as $40 \%$ per annum and the strike price ranges from $\$ 85$ to $\$ 115$. We note that when the strike price $K=\$ 110, \$ 115$, the relative differences are more than $100 \%$ and $200 \%$, respectively.

Panel C illustrates the differences in option prices with different maturities. For the at-the-money options considered, the relative differences are rather consistent even when maturity increases to 6-month $(T=126)$ and 1year $(T=252)$.

Panel D illustrates the effect of daily price limits on option prices, where $\delta$ is set to values in a range of $1 \%$ to $10 \%$. As expected, the relative difference increases as the absolute price limit is lower. For the out-of-themoney option considered (strike price $K=105$ ), the relative difference in option price with restriction and without restriction is more than $30 \%$ when the price limit is set as $5 \%$.

\section{Conclusions}

In practice, exchanges often set daily limits for the price changes of traded assets. These restrictions have a direct impact on prices of options traded on these assets since they rule out dramatic changes in asset prices. In this paper, we derive closed-form solution of option pricing formula with restrictions on underlying asset price changes. Using numerical examples, we illustrate that very often the impact of such restrictions on option prices can be substantial.

\section{Acknowledgements}

The research of Lei Shi is supported by NSFC (11161053).

\section{References}

[1] F. Black and M. Scholes, "The Pricing of Options and Corporate Liabilities," Journal of Political Economy, Vol. 81, No. 3, 1973, pp. 637-654. doi:10.1086/260062

[2] R. C. Merton, "Theory of Rational Option Pricing," Bell Journal of Economics, Vol. 4, No. 1, 1973, pp. 141-183. doi: $10.2307 / 3003143$

[3] J. C. Hull, "Options, Futures, and Other Derivatives," 8th Edition, Prentice Hall, Upper Saddle River, 2011.

[4] S. L. Heston, "A Closed-Form Solution for Options with Stochastic Volatility with Applications to Bond and Currency Options," Review of Financial Studies, Vol. 6, No. 2, 1993, pp. 327-343. doi:10.1093/rfs/6.2.327 


\section{Appendix}

In the appendix, we first derive the moment generating function (mgf), and characteristic function (CF) of a truncated normal random variable. Recall that the pdf of a truncated normal random variable $X \sim \mathcal{N}_{\text {trc }}\left(\mu, \sigma^{2}\right)$,

$$
f_{\mathrm{trc}}(x)=\frac{1}{c} f(x), \quad x \in[\mu-a, \mu+b],
$$

where $f(x)$ is the normal density function and

$$
c=\Phi\left(\frac{b}{\sigma}\right)+\Phi\left(\frac{a}{\sigma}\right)-1 .
$$

The mgf of a truncated normal random variable as $X \sim \mathcal{N}_{\text {trc }}\left(\mu, \sigma^{2}\right)$ is derived as,

$$
\begin{aligned}
M_{\text {trc }}(\omega) & =E\left[\mathrm{e}^{\omega X}\right]=\int_{\mu-a}^{\mu+b} \mathrm{e}^{\omega x} f_{\text {trc }}(x) \mathrm{d} x \\
= & \int_{\mu-a}^{\mu+b} \mathrm{e}^{\omega x} \frac{1}{\sqrt{2 \pi} \sigma c} \mathrm{e}^{-\frac{(x-\mu)^{2}}{2 \sigma^{2}}} \mathrm{~d} x \\
= & \frac{1}{c} \mathrm{e}^{\mu \omega+\frac{1}{2} \sigma^{2} \omega^{2}} \int_{\mu-a}^{\mu+b} \frac{1}{\sqrt{2 \pi} \sigma} \mathrm{e}^{-\frac{\left[x-\left(\mu+\sigma^{2} \omega\right)\right]^{2}}{2 \sigma^{2}}} \mathrm{~d} x \\
= & \frac{c^{\prime}(\omega)}{c} M(\omega)
\end{aligned}
$$

where $M(\omega)=\mathrm{e}^{\mu \omega+\frac{1}{2} \sigma^{2} \omega^{2}}$ is the mgf of a normal random variable, and

$$
\begin{aligned}
& c^{\prime}(\omega)=\int_{\mu-a}^{\mu+b} \frac{1}{\sqrt{2 \pi} \sigma} \mathrm{e}^{-\frac{\left[x-\left(\mu+\sigma^{2} \omega\right)\right]^{2}}{2 \sigma^{2}}} \mathrm{~d} x \\
& =\int_{-\infty}^{\mu+b} \frac{1}{\sqrt{2 \pi} \sigma} \mathrm{e}^{-\frac{\left[x-\left(\mu+\sigma^{2} \omega\right)\right]^{2}}{2 \sigma^{2}}} \mathrm{~d} x-\int_{-\infty}^{\mu-a} \frac{1}{\sqrt{2 \pi} \sigma} \mathrm{e}^{-\frac{\left[x-\left(\mu+\sigma^{2} \omega\right)\right]^{2}}{2 \sigma^{2}}} \mathrm{~d} x \\
& =\Phi\left(\frac{\mu+b-\left(\mu+\sigma^{2} \omega\right)}{\sigma}\right)-\Phi\left(\frac{\mu-a-\left(\mu+\sigma^{2} \omega\right)}{\sigma}\right) \\
& =\Phi\left(\frac{b}{\sigma}-\sigma \omega\right)+\Phi\left(\frac{a}{\sigma}+\sigma \omega\right)-1 .
\end{aligned}
$$

Similarly, the characteristic function (CF) of a truncated normal random variable $X \sim \mathcal{N}_{\text {trc }}\left(\mu, \sigma^{2}\right)$ is given by,

$$
\psi_{\mathrm{trc}}(\omega)=M_{\mathrm{trc}}(i \omega)=\frac{c^{\prime}(i \omega)}{c} \psi(\omega),
$$

where $c$ is given by (10), and

$$
\psi(\omega)=\mathrm{e}^{\mu i \omega-\frac{1}{2} \sigma^{2} \omega^{2}},
$$

is the $\mathrm{CF}$ of a normal random variable,

$$
c^{\prime}(i \omega)=\Phi\left(\frac{b}{\sigma}-\sigma i \omega\right)+\Phi\left(\frac{a}{\sigma}+\sigma i \omega\right)-1 .
$$

Next, we derive the CF of the log price with truncated distribution. Denote $Z=X_{1}+\cdots+X_{T}$ as the sum of the iid sequence $X_{1}, \cdots, X_{T} \sim \mathcal{N}_{\text {trc }}\left(\mu, \sigma^{2}\right)$, then the $\mathrm{mgf}$ of $Z$ is

$$
M_{Z}(\omega ; \mu, \sigma, a, b)=\left[M_{\mathrm{trc}}(\omega)\right]^{T}=\left[\frac{c^{\prime}(\omega)}{c} M(\omega)\right]^{T},
$$

and the $\mathrm{CF}$ of $Z$ is

$$
\psi_{Z}(\omega ; \mu, \sigma, a, b)=\left[\psi_{\text {trc }}(\omega)\right]^{T}=\left[\frac{c^{\prime}(i \omega)}{c} \psi(\omega)\right]^{T},
$$

where we emphasize that the characteristic function $\psi_{Z}$ also depends on normal random variable parameters $\mu, \sigma$ and truncation parameters $a, b$.

Denote $S_{t}$ as the stock price at the end of day $t$ and $X_{t}=\ln S_{t}$ is the $\log$ price. Suppose we have $T$ trading days, $1, \cdots, T$, and the daily log returns are iid truncated normally distributed in a risk-neutral world, i.e., $Y_{t} \sim$ $\mathcal{N}_{\text {trc }}\left(\theta, \sigma^{2}\right),\left(Y_{t}=\ln S_{t}-\ln S_{t-1}, t=1, \cdots, T\right)$. The log price at the end of day $T$ is

$$
X_{T}=X_{0}+\sum_{t=1}^{T} Y_{t}, Y_{t} \sim \operatorname{iid} \mathcal{N}_{\text {trc }}\left(\theta, \sigma^{2}\right) .
$$

The mgf and CF of $X_{T}$ are derived as

$$
\begin{aligned}
& M_{X_{T}}(\omega)=E\left[\mathrm{e}^{\omega X_{T}}\right]=\mathrm{e}^{\omega X_{0}}\left[\frac{c^{\prime}(\omega)}{c} M(\omega)\right]^{T}, \\
& \psi_{X_{T}}(\omega)=E\left[\mathrm{e}^{i \omega X_{T}}\right]=\mathrm{e}^{i \omega X_{0}}\left[\frac{c^{\prime}(i \omega)}{c} \psi(\omega)\right]^{T},
\end{aligned}
$$

where

$$
\begin{gathered}
c=\Phi\left(\frac{b}{\sigma}\right)+\Phi\left(\frac{a}{\sigma}\right)-1, \\
c^{\prime}(\omega)=\Phi\left(\frac{b}{\sigma}-\sigma \omega\right)+\Phi\left(\frac{a}{\sigma}+\sigma \omega\right)-1, \\
M(\omega)=\mathrm{e}^{\theta \omega+\frac{1}{2} \sigma^{2} \omega^{2}}, \text { normal mgf } \\
\psi(\omega)=\mathrm{e}^{\theta i \omega-\frac{1}{2} \sigma^{2} \omega^{2}}, \text { normal CF. }
\end{gathered}
$$

